\title{
Maternidade, paternidade e vida acadêmica: impactos e percepções de mães e pais estudantes de medicina
}

\section{Maternity, paternity and academic life: impacts and views of parents who are studying medicine}

\author{
Quécia Hosana Fatel Brito' (D) quecia_fatelb@outlook.com \\ Katia de Miranda Avena ${ }^{2}$ (D) katiaavena@hotmail.com \\ Evelise Maria Labatut Portilho ${ }^{3}$ (D) eveliseportilho@gmail.com \\ Mariana Araújo Pereira ${ }^{4}$ (D) araujopereira.mariana@gmail.com \\ Luiz Fernando Quintanilha ${ }^{1,2,3}$ (D) quintanilha.lf@gmail.com
}

\begin{abstract}
RESUMO
Introdução: Aspectos pessoais, como a maternidade e a paternidade podem representar grandes desafios acadêmicos e profissionais, uma vez que requerem tempo, envolvimento afetivo, atenção e cuidado. Principalmente no curso de Medicina, caracterizado pela extensa rotina de estudos e carga horária, essa conjuntura pode ser ainda mais impactante.

Objetivo: Este estudo teve como objetivos avaliar o impacto da maternidade/paternidade no rendimento acadêmico de estudantes de Medicina, e analisar as principais percepções, motivações e desafios deles durante a formação médica.

Método: Trata-se de um estudo transversal, quanti-qualitativo de abordagem descritiva e analítica que avaliou o perfil e as percepções de 85 mães/pais estudantes de Medicina em todo o país. Como instrumento de coleta, foi empregado um formulário virtual, semiestruturado, contendo questões sociodemográficas de única escolha e de percepções utilizando a escala Likert. A fim de avaliar o impacto da maternidade/paternidade no rendimento acadêmico, parte dos respondentes de uma das instituições avaliadas teve seus rendimentos acadêmicos obtidos para a comparação com amostras aleatórias e não identificadas de estudantes sem filhos da mesma instituição.

Resultado: Os estudantes possuem clareza e não se arrependem de suas escolhas pessoais (93\%), têm orgulho de ser mãe/pai universitários (93\%) e ressaltam a importância e essencialidade da rede de apoio (88\%). Ao serem questionados quanto ao rendimento acadêmico, as mães e os pais estudantes de Medicina expressaram limitação na compatibilidade dos afazeres e responsabilidades pessoais e profissionais (42\%) e relataram que as obrigações parentais comprometem o rendimento no curso de Medicina (53\%). Neste âmbito, porém, nossos dados apontam para um rendimento acadêmico de mães e pais similar aos de estudantes sem filhos.

Conclusão: Os estudantes de Medicina com filhos, apesar de relatarem limitação na capacidade de associar os afazeres e as responsabilidades pessoais e acadêmicas, não se arrependem das suas escolhas e têm orgulho de exercer a dupla função. Além disso, eles apresentam rendimento acadêmico semelhante ao de estudantes sem filhos, apesar de acreditarem que as obrigações da maternidade/paternidade comprometem o seu rendimento acadêmico. Destacam-se a essencialidade da rede de apoio para realização das atividades acadêmicas e a uniformidade dessas percepções entre variáveis como sexo, estado civil e ciclo acadêmico.
\end{abstract}

Palavras-chave: Educação de Graduação em Medicina; Educação Médica; Estudantes de Medicina; Planejamento Familiar; Saúde do Estudante.

\section{ABSTRACT}

Introduction: Personal aspects such as maternity and paternity can represent major academic and professional challenges, as they require time, emotional involvement, attention and care. Mainly in the medical schools, characterized by the extensive study routine and workload, this situation can be even more impactful.

Objective: To assess the impact of parenthood on the academic performance of medical students, as well as to analyze their main views, motivations and challenges during medical training.

Method: Cross-sectional, quantitative-qualitative study with a descriptive and analytical approach that evaluated the profile and perceptions of 85 parents who are studying medicine in Brazil. A semi-structured virtual questionnaire was used containing single-choice sociodemographic questions and evaluation of views using the Likert scale. In order to assess the impact of parenthood on academic performance, the academic performance of some of the respondents from one of the institutions evaluated was considered for comparison with random and unidentified samples of childless students from the same institution. Result: Students are clear and do not regret their personal choices (93\%), are proud to be parent students (93\%) and emphasize the importance and essentiality of the support network (88\%). When asked about academic performance, the parents who are medical students express limitations in the compatibility of personal and professional responsibilities (42\%) and report that parental duties compromise their performance at medical school (53\%). In this context, however, our data indicate that the academic performance of mothers and fathers is similar to that of childless students.

Conclusion: Medical students with children, despite reporting limitations in the ability to combine personal and academic responsibilities, do not regret their choices and are proud to exercise both roles. Furthermore, their academic performance is similar to that of childless students, despite believing that parental duties compromise their academic performance. They highlight the essential role played by the support network for performing academic activities and these perceptions between variables such as gender, marital status and academic cycle are distinctively uniform.

Keywords: Undergraduate Medical Training; Medical Education; Medical Students; Family Planning; Student Health.

1 Universidade Salvador, Salvador, Bahia, Brasil.

2 Centro Universitário UniFTC, Salvador, Bahia, Brasil.

${ }^{3}$ Pontifícia Universidade Católica do Paraná, Curitiba, Paraná, Brasil.

${ }^{4}$ Instituto Gonçalo Moniz, Salvador, Bahia, Brasil.

Editora-chefe: Rosiane Viana Zuza Diniz. | Editor associado: Pedro Tadao Hamamoto Filho.

Recebido em 23/07/21; Aceito em 25/10/21. | Avaliado pelo processo de double blind review. 


\section{INTRODUÇÃO}

O curso de graduação em Medicina se destaca há anos como um dos mais procurados e concorridos do país ${ }^{1}$. Se o acesso já apresenta desafios, a permanência no curso médico e a conclusão dele exigem dedicação, determinação e esforço em razão da extensa carga horária e da longa rotina de estudos, que geram, por vezes, sobrecarga cognitiva e emocional ${ }^{2-6}$. O cotidiano vivenciado na graduação sugere o contato com realidades complexas, mudanças e adaptações no estilo de vida, o que resulta, muitas vezes, em sintomas de estresse e ansiedade ${ }^{7}$. Os estudantes são expostos a momentos de exaustão, privação do sono, autocobrança e eventos negativos de medo e angústia durante o atendimento clínico com os pacientes, além de dor e sofrimento provocados pelo íntimo contato com os casos de enfermidade e morte humanas ${ }^{8,9}$.

Existem, ainda, aspectos pessoais que podem impactar o processo acadêmico e provocar diversas consequências no desempenho do estudante de Medicina. Um desses aspectos é a maternidade/paternidade que, além de impactar economicamente, pode representargrandes desafios acadêmicos e profissionais ${ }^{10}$. Especialmente na Medicina, cuja formação pode durar até 11 anos, considerando a residência médica, a condição de maternidade/paternidade pode representar um desafio para o estudante, exigindo muita disposição, esforço e adaptação. Considera-se não somente a questão biológica, mas também circunstâncias que demandam atenção, cuidado, envolvimento afetivo, recursos financeiros e tempo dedicado à criança ${ }^{10}$. Nesse cenário, devido ao receio de que tais demandas possam comprometer os processos acadêmicos e profissionais, é relatado que muitas mulheres adiam ao máximo a maternidade ${ }^{11,12}$.

Diante do exposto, compreender os impactos da maternidade e paternidade no desempenho acadêmico é essencial tanto para a adequada programação e decisão dos estudantes quanto para professores e gestores lidarem melhor com esses casos, tornando o momento da graduação em Medicina mais amigável e harmônico. Apesar da importância dessa temática, são escassas as pesquisas com delineamento metodológico analítico adequado que suportem discussões cientificamente aprofundadas.

Nesse sentido, o objetivo deste estudo foi avaliar o impacto da maternidade/paternidade no rendimento acadêmico de estudantes de Medicina do Brasil, bem como analisar as principais percepções, motivações e desafios vivenciados por pais e mães estudantes universitários.

\section{MÉTODOS}

Trata-se de um estudo transversal, de natureza quantiqualitativa e abordagem descritiva e analítica, que avaliou 85 estudantes maiores de 18 anos, com filhos e devidamente matriculados em cursos de Medicina de instituições de ensino superior (IES) reconhecidas pelo Ministério da Educação (MEC) em todo o país, no período de outubro de 2020 a janeiro de 2021.

Os estudantes foram convidados a participar da pesquisa por meio do método snowball, técnica de amostragem não probabilística cujos sujeitos de estudo recrutam outros sujeitos futuros entre seus conhecidos. Como instrumento de coleta, empregou-se um formulário virtual, semiestruturado, por meio da ferramenta Google Forms, contendo questões sociodemográficas de única escolha e de percepções utilizando a escala Likert com cinco níveis de respostas, sendo considerados três estratos para as análises: concordância (total ou parcial), indiferença e discordância (total ou parcial).

Avaliaram-se parâmetros como sexo, idade, local de residência, quantidade de filhos, possível rede de apoio, ciclo acadêmico (básico - do primeiro ao quarto semestre; clínico - do quinto ao oitavo semestre; internato - do nono ao $12^{\circ}$ semestre) e percepções sobre a maternidade/paternidade no decorrer da graduação em Medicina.

A fim de avaliar o impacto da maternidade/paternidade no rendimento acadêmico, parte dos respondentes de uma das instituições avaliadas ( $n=15)$, após a anuência da coordenação acadêmica, teve seus rendimentos acadêmicos (escore) obtidos para a comparação com amostras aleatórias e não identificadas de estudantes sem filhos da mesma instituição. Os participantes do grupo de interesse da pesquisa (mães/pais) estavam cursando diferentes semestres da graduação. Por isso, para comparar o desempenho com os estudantes sem filhos de uma forma mais robusta, utilizaram-se amostras aleatórias de estudantes em cinco semestres distintos: terceiro, quinto, oitavo, nono e $12^{\circ}$ ( $n=15$ em cada um desses grupos).

Os dados foram tabulados no Programa Excel e analisados pelo Programa GraphPad Prism 8.0. Inicialmente, realizou-se o teste de normalidade Shapiro-Wilk que indicou uma distribuição normal dos dados. Assim, para os dados categóricos, utilizamos o teste Qui-quadrado ou Fisher. Para os dados contínuos, foram realizados testes de comparação t-Student par a par (mães/pais versus sem filhos para cada semestre). Consideraram-se valores de $p<0,05$ como estatisticamente significantes.

Todos os participantes do estudo foram esclarecidos sobre os objetivos e a metodologia da pesquisa, sendo garantido o sigilo sobre a origem dos dados. A participação voluntária se concretizou por meio da assinatura virtual do Termo de Consentimento Livre e Esclarecido (TCLE). O presente trabalho está em consonância com a Resolução n 466/2012 do Conselho Nacional de Saúde (CNS) e foi aprovado pelo Comitê de Ética em Pesquisa do Instituto Mantenedor de Ensino Superior da Bahia - Certificado de Apresentação para Apreciação Ética (CAAE) n 38691820.4.0000.5032. 


\section{RESULTADOS}

\section{Características da população}

A amostra do presente estudo foi composta predominantemente por estudantes do sexo feminino $(78,8 \%)$, de cores branca $(50,6 \%)$ e parda $(38,8 \%)$, com média de idade de $31,2 \pm 6,3$ anos, casados $(68,2 \%)$ e que possuem apenas um filho $(71,8 \%)$. Apesar de a maioria dos estudantes residir em estados da Região Nordeste (52,9\%), nosso estudo teve uma abrangência nacional, contemplando estudantes de todas as regiões do país (Gráfico 1).

\section{Percepção dos estudantes quanto ao desempenho acadêmico}

Acerca das percepções das mães e dos pais estudantes de Medicina sobre o impacto da maternidade/paternidade na vida acadêmica, identificamos que a população avaliada possui orgulho de ser mãe/pai universitário(a) (Gráfico 2A) e não se arrepende de suas escolhas pessoais (Gráfico 2B), entretanto destacamos a essencialidade da rede de apoio para permitir o desenvolvimento de suas atividades acadêmicas (Gráfico 2C). Contudo, ao serem questionados quanto ao desempenho acadêmico, os respondentes relataram limitação na capacidade de desenvolver satisfatoriamente os afazeres e as responsabilidades dos dois papéis (Gráfico 2D) e expuseram que as obrigações da maternidade/paternidade comprometem o rendimento no curso de Medicina (Gráfico 2E).

Além da avaliação descritiva, foram realizados testes de associação entre as variáveis estudadas. Houve uma associação estatisticamente significante entre o número de filhos (dicotomizado em "um filho" ou "dois ou mais") e

Gráfico 1.Características da população estudada $(n=85)$. (A) Variáveis categóricas. (B) Idade em anos. (C) Abrangência do estudo de acordo com a região do país.

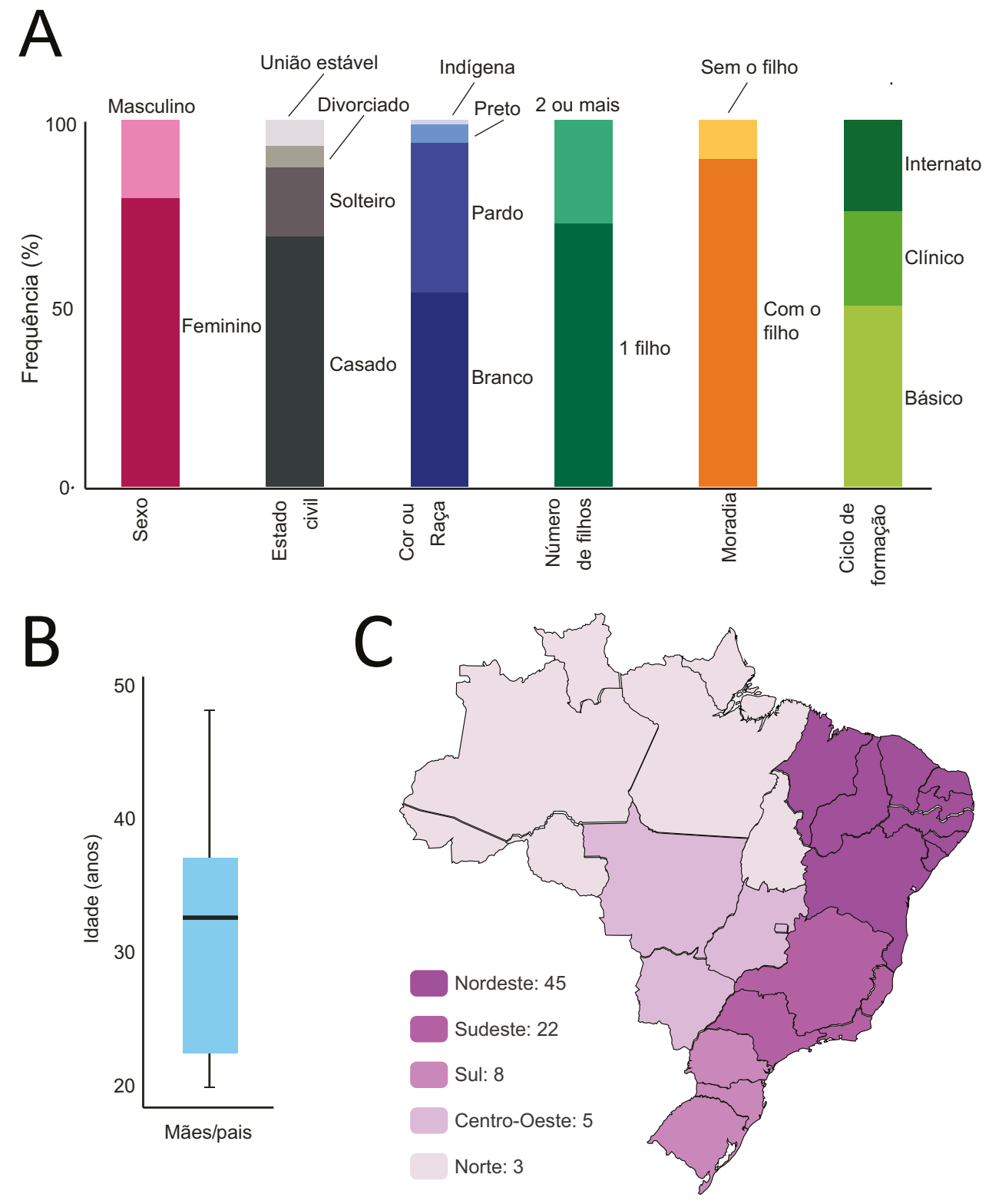


Gráfico 2.Distribuição das percepções dos pais/das mães estudantes de Medicina em relação ao impacto da paternidade/ maternidade na vida acadêmica $(n=85)$.

Concordo Indiferente Discordo

A)

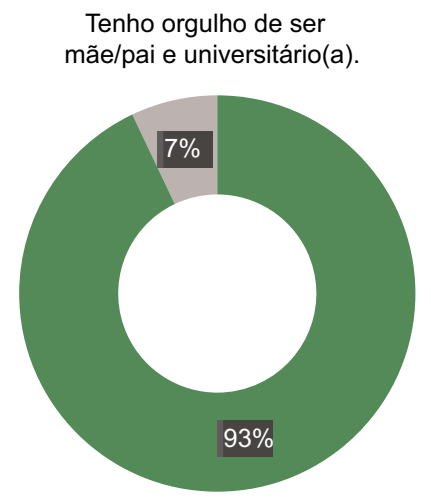

C)

Se não fosse a minha rede de apoio, eu não estaria conseguindo conciliar a maternidade/paternidade e a vida acadêmica.

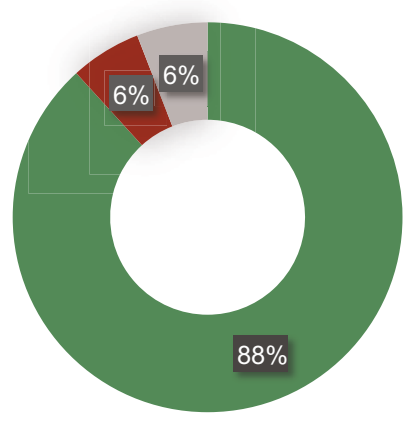

D)

Sinto que não estou sendo nem bom pai/boa mãe, nem bom/boa estudante.

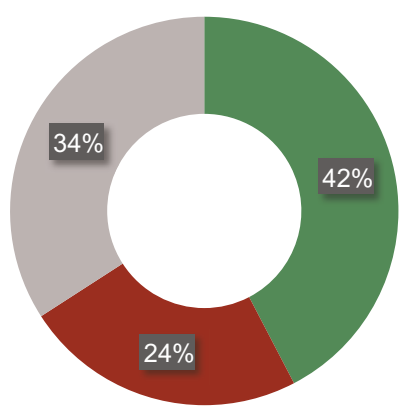

B)

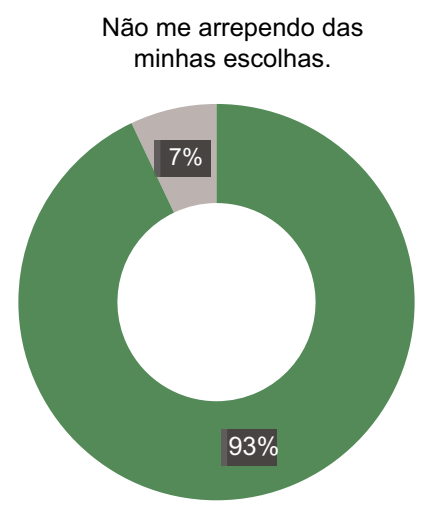

E)

As minhas obrigações como pai/mãe atrapalham meu rendimento.

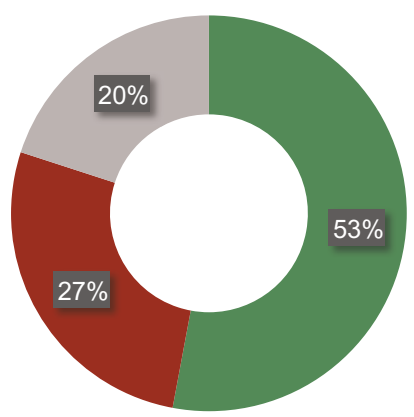

a percepção de que "Se não fosse a minha rede de apoio, eu não estaria conseguindo conciliar a maternidade/paternidade e a vida acadêmica". Nesse caso, houve maior concordância dos estudantes que possuem apenas um filho quando confrontados com aqueles com dois ou mais $(91,8 \%$ versus $79,2 \%$, respectivamente, $p=0,0289$ ).

Ao analisarmos alguns dos relatos, fomos capazes de inferir que o planejamento familiar em virtude da primeira gestação pode contribuir para a conciliação dos afazeres domésticos e acadêmicos. Essa percepção é evidenciada por falas como "A maturidade que eu adquiri com meu primeiro filho foi fundamental para que desse um passo rumo a essa nova vida acadêmica" e "Há impactos positivos como saber utilizar melhor o tempo e procrastinar menos". Entretanto, notou-se eventual insatisfação com a falta de ações afirmativas institucionais, como pudemos observar no seguinte trecho:"Fui muito julgada por escolher engravidar na faculdade e não tive apoio da instituição. Foram-me negados direitos e sugeriram que eu trancasse a faculdade".

Com relação às demais variáveis, não foram encontradas associações entre as percepções analisadas neste trabalho, demonstrando uniformidade entre os estudantes, independentementede suas características, quandoquestionados sobre os impactos dos filhos na sua formação médica.

\section{Impacto da maternidade/paternidade no rendimento acadêmico}

A percepção majoritária dos estudantes é de que as obrigações parentais prejudicam o rendimento acadêmico, o que foi enfatizado em falas como:"temos uma responsabilidade muito grandena formação dos nossos filhos [...]. Por isso, acredito que haja um impacto na vida de acadêmicos, principalmente de Medicina pelas demandas que nos são cobradas diariamente". Ao comparar os rendimentos acadêmicos, foi observado que 
Gráfico 3. Rendimentos acadêmicos (escores) dos estudantes de Medicina com filhos provenientes de diferentes semestres $(n=15)$ em comparação a estudantes sem filhos escolhidos randomicamente em cinco semestres $(n=15$ para cada grupo randômico).

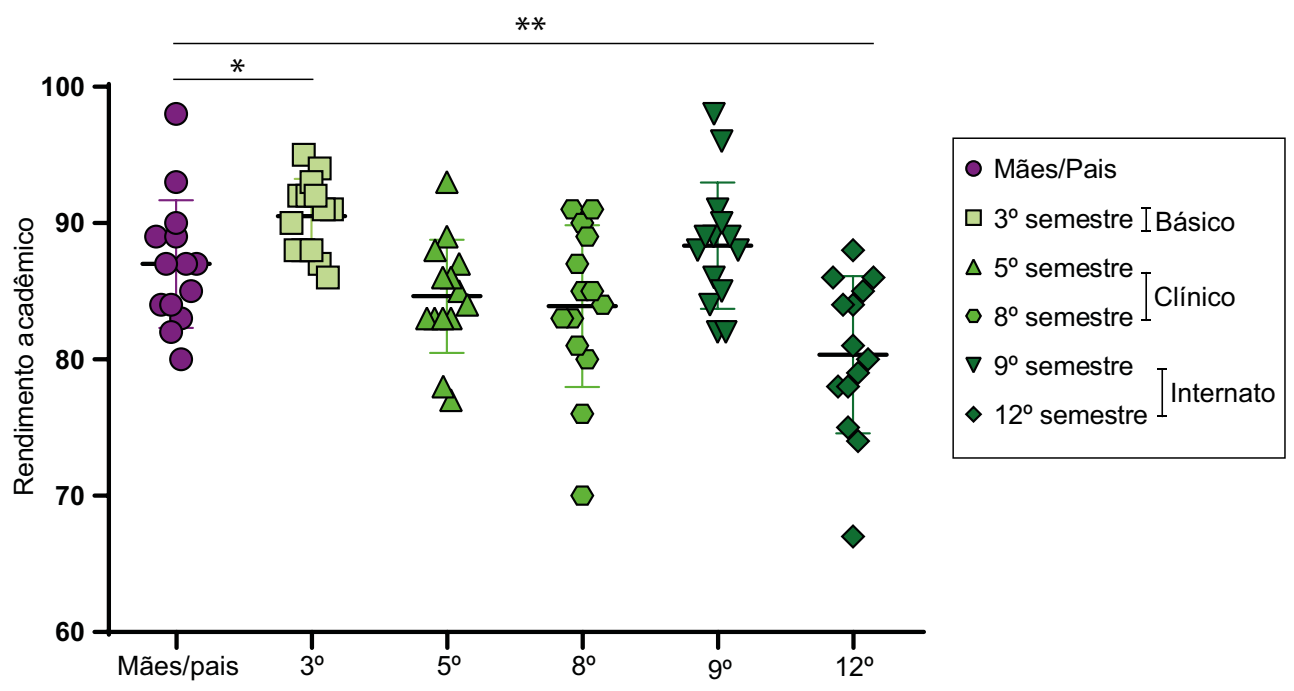

no ciclo básico, aqui representado pelo terceiro semestre, e no final do internato, representado pelo $12^{\circ}$ semestre, houve uma diferença entre os escores daqueles que possuem e não possuem filhos. Nossos dados demonstraram que os estudantes com filhos obtiveram um menor rendimento acadêmico no ciclo básico $(87,0$ versus 90,$5 ; \mathrm{p}=0,02)$, entretanto esse cenário se inverteu quando se analisou o final do internato $(87,0$ versus 80,4; $p=0,002$ ) (Gráfico 3). É importante citar que, no ciclo clínico (quinto e oitavo semestres) e no início do internato (nono semestre), não foram encontradas diferenças estatisticamente significantes entre os escores.

\section{DISCUSSÃO}

Nossa amostra de respondentes foi constituída, sobretudo, por estudantes do sexo feminino e de cores branca e parda. Apesar de possivelmente termos mais engajamento feminino em pesquisas dessa temática, essas características estão de acordo com as tendências apontadas pelo censo dos estudantes de Medicina. Este descreve um recente fenômeno de feminização do curso, bem como aponta para um aumento expressivo de vagas preenchidas por estudantes negros e pardos ${ }^{13}$. O perfil dos respondentes, além de apresentar características semelhantes ao censo supracitado, possui representação geográfica abrangente, fortalecendo a relevância deste estudo em âmbito acadêmico nacional.

Todavia, podemos apontar algumas peculiaridades associadas à idade e ao estado civil que não se aplicam ao padrão nacional dos estudantes do ensino superior. De maneira contrária, nossa amostra contemplou estudantes mais velhos e casados, em contraste com padrão nacional ${ }^{4,14,15}$. Isso pode ser explicado pela temática do trabalho e pelos critérios de inclusão aplicados, os quais objetivaram avaliar apenas mães e pais estudantes, diferenciando-os da média nacional de idade (de 19 a 24 anos) e estado civil (solteiros).

Nossos resultados demonstram que, apesar de estudos indicarem uma tendência ao adiamento da maternidade/ paternidade em função de um projeto de vida orientado para o acadêmico e o profissional (por exemplo: busca por estabilidade financeiraeconsolidação da carreiranomercado de trabalho) ${ }^{16-19}$, há uma percepção hegemônica entre os participantes em relação ao orgulho e à clareza de suas escolhas. Nesse contexto, apesar de os nossos resultados não apresentarem diferenças significativas nas percepções entre homens e mulheres, um estudo realizado em três cursos médicos suíços demonstrou que as mulheres médicas apresentam, em geral, menores índices de empregabilidade e possuem qualificações menos avançadas, ao mesmo tempo que apresentam um estilo de vida mais direcionado aos aspectos relacionados à maternidade ${ }^{20}$. É, entretanto, importante levarmos em consideração as diferenças culturais e socioeconômicas entre os países, as quais podem influenciar as opções profissionais. Do mesmo modo, a escassez de estudos nacionais nessa temática contribui para a dificuldade na realização de comparações entre os achados.

Além do já citado fenômeno da feminização da Medicina, outro aspecto importante a ser discutido é a busca cada vez mais aparente por um estilo de vida mais saudável e moderno. Em um estudo na Universidade de Zurique com 526 médicos, itens como "creches" e "empregos em tempo parcial" foram elencados como importantes aspectos a serem instituídos nos seus locais de trabalho ${ }^{21}$.

Com relação à importância da rede de apoio, houve um grau de concordância maior na amostra representada 
por estudantes com apenas um filho. Apesar de acreditarmos que um maior número de filhos demandaria uma maior necessidade dessa rede, é possível que a inexperiência de mães/pais de "primeira viagem" tenha contribuído para esse achado. A necessidade da rede de apoio está associada ao planejamento, à organização e à divisão de afazeres que os pais precisam desenvolver para conciliar as questões acadêmicas e pessoais. Esse duplo papel proporciona o desenvolvimento de estratégias para conciliação de ambos, ressaltando as divisões na estrutura familiar das atividades do cotidiano, com a finalidade de minimizar a sobrecarga dos pais ${ }^{22}$.

Ainda nesse contexto, um estudo realizado na Faculdade de Medicina da Universidade Federal de Minas Gerais (UFMG) destacou a preocupação dos estudantes com a compatibilização dos aspectos profissionais e familiares, ressaltando a importância e a dificuldade de conciliar maternidade e profissão ${ }^{23}$. Aparentemente, na trajetória desses estudantes, essa rede de apoio contribui para um melhor planejamento da complexa situação vivenciada, dinamizando o tempo para estudos, quer seja no ambiente acadêmico ou doméstico. Além disso, sabe-se que a renda mensal familiar dos estudantes de Medicina é consideravelmente superior à média da população ${ }^{24}$, o que os coloca em uma possível situação social privilegiada, de modo que essa condição pode ser um fator atenuador dos impactos acadêmicos da maternidade/paternidade.

Mesmo com esses possíveis privilégios, os nossos dados apontam para uma autopercepção de impacto negativo no rendimento acadêmico, devido às responsabilidades inerentes da maternidade/paternidade. Entretanto, três das cinco comparações realizadas apontam para um rendimento acadêmico de mães e pais similar aos de estudantes sem filhos, indicando que a maternidade/paternidade não está associada, possivelmente, a diferenças marcantes em escores obtidos nas avaliações de aprendizagem realizadas durante a formação médica.

O presente trabalho lança luz a um tema ainda pouco estudado no Brasil e que deveria ser mais explorado, em razão 1. da crescente feminização do curso de Medicina no país, 2. das características sociais e biológicas que recaem especialmente sobre as mulheres, 3. dos obstáculos inerentes à conciliação de vida familiar, criação dos filhos, estudos e trabalho, e 4. das diferenças de gênero relacionadas aos cuidados com os filhos e as expectativas de carreira. Aqui, são avaliadas as percepções, além de dados de rendimento acadêmico de mães e pais estudantes de Medicina de todo o país, contribuindo para essa temática de maneira substancial, o que pode refletir na quebra de paradigmas e em melhores práticas na gestão da educação médica em todo o país.
Ao longo do percurso metodológico, não foram investigados aspectos como planejamento familiar e a decisão de conceber filho(s) durante a graduação em Medicina. Aqui, avaliamos o impacto da maternidade/paternidade no rendimento acadêmico do estudante, independentemente de tal planejamento, bem como não foi pretendido identificar os atores principais da rede de apoio, somente a existência ou não desse vínculo. Ademais, em virtude do contexto pandêmico da coronavirus disease 2019 (Covid-19), optamos por uma técnica de amostragem não probabilística, o que pode limitar algumas das inferências levantadas por este estudo. Sendo assim, a realização de novas pesquisas considerando esses e outros aspectos, como a categoria administrativa da IES e a existência de ações afirmativas (como mentorias) para estes estudantes, é de suma importância para melhor aprofundamento nessa temática. Além disso, pelo que sabemos, nenhum outro estudo investigou de forma analítica e com abrangência nacional as condições vivenciadas por pais e mães estudantes de Medicina no país, o que corrobora a originalidade e importância dos resultados aqui apresentados.

\section{CONCLUSÃO}

Os estudantes de Medicina com filhos, apesar de relatarem limitação na capacidade de associar satisfatoriamente os afazeres e as responsabilidades pessoais e acadêmicas, sentem-se orgulhosos por exercerem essa dupla função. Além disso, eles apresentam rendimento acadêmico semelhante ao de estudantes sem filhos, apesar de acreditarem que as obrigações da maternidade/paternidade comprometem o seu rendimento acadêmico. É destacada a essencialidade da rede de apoio para realização das atividades acadêmicas, e nota-se uniformidade das percepções ao compararmos variáveis como sexo, estado civil e ciclo acadêmico.

\section{CONTRIBUIÇÃO DOS AUTORES}

Quécia Hosana Fatel Brito participou da coleta e interpretação de dados, e da redação final do artigo. Katia de Miranda Avena e Evelise Maria Labatut Portilho participaram da concepção do projeto e da revisão do texto. Mariana Araújo Pereira participou da análise estatística e da redação final do artigo. Luiz Fernando Quintanilha participou da concepção do projeto, da coleta e interpretação de dados, da análise estatística e da redação final do artigo.

\section{CONFLITO DE INTERESSES}

Declaramos não haver conflito de interesses.

\section{FINANCIAMENTO}

Declaramos não haver financiamento. 


\section{REFERÊNCIAS}

1. Brasil. Inscritos no $2^{\circ}$ Sisu 2020 já podem conferir se foram selecionados. Sisu; 2020 [acesso em 20 mar 2021]. Disponível em: https://www.gov. $\mathrm{br} / \mathrm{mec} / \mathrm{pt}$-br/assuntos/noticias/inscritos-no-2o-sisu-2020-ja-podemconferir-se-foram-selecionados.

2. Steiner-Hofbauer V, Holzinger A. How to cope with the challenges of medical education? Stress, depression, and coping in undergraduate medical students. Acad Psychiatry. 2020;44:380-7. doi: 10.1007/s40596020-01193-1.

3. Benevides-Pereira AMT, Gonçalves MB. Transtornos emocionais e a formação em Medicina: um estudo longitudinal. Rev Bras Educ Med. 2009;33(1):10-23 [acesso em 13 mar 2021]. Disponível em: http://www.scielo.br/scielo.php?script=sci_arttext\&pid=S0100$55022009000100003 \&$ lng $=e n \& n r m=i s o \& t$ lng $=$ pt.

4. Fiorotti KP, Rossoni RR, Miranda AE. Perfil do estudante de Medicina da Universidade Federal do Espírito Santo, 2007. Rev Bras Educ Med. 2010;34(3):355-62 [acesso em 20 mar 2021]. Disponível em: http://www.scielo.br/scielo.php?script=sci_arttext\&pid=S0100$55022010000300004 \&$ lng=en\&nrm=iso\&tlng=pt.

5. Pacheco JPG, Giacomin HT, Tam WW, Ribeiro TB, Arab C, Bezerra IM, et al. Mental health problems among medical students in Brazil: a systematic review and meta-analysis. Rev Bras Psiquiatr. 2017;39(4):369-78 [acesso em 20 mar 2021]. Disponível em: http://www.scielo.br/scielo.php?script=sci_ arttext\&pid=S1516-44462017000400369\&Ing=en\&nrm=iso\&tlng=en.

6. Santos VH, Ferreira JH, Alves GCA, Naves NM, Oliveira SL, Raimondi GA, et al. Currículo oculto, educação médica e profissionalismo: uma revisão integrativa. Interface Comun Saúde Educ. 2020;24:e190572 [acesso em 8 jul 2021]. Disponível em: https://www.interface.org.breissn1807-5762.

7. Moutinho ILD, Maddalena NCP, Ronald RK, Lucchetti ALG, Tibiriçá SHC, Ezequiel OS, et al. Depression, stress and anxiety in medical students: a cross-sectional comparison between students from different semesters. Rev Assoc Med Bras. 2017;63(1):21-8. doi: 10.1590/1806-9282.63.01.21.

8. Dyrbye LN, Thomas MR, Shanafelt TD. Systematic review of depression, anxiety, and other indicators of psychological distress among U.S. and Canadian medical students. Acad Med. 2006;81(4):354-73 [acesso em 13 mar 2021]. Disponível em: https://pubmed.ncbi.nlm.nih.gov/16565188/.

9. Feodrippe ALO, Brandão MCF, Valente TCO. Qualidade de vida de estudantes de Medicina: uma revisão. Rev Bras Educ Med. 2013;37(3):41828 [acesso em 21 mar 2021]. Disponível em: http://www.scielo.br/scielo. php?script=sci_arttext\&pid=S0100-55022013000300014\&lng=en\&nrm=i so\&tlng=pt.

10. Ferreira MGAM, Lopez-Gómez J, David HMSL, Navalon-Mira A, Brunherotti MAA, Gea-Caballero V, et al. Profile of young university students and their perceptions of motherhood and fatherhood. Rev Enferm Ref. 2019;2019(23):81-8.

11. Ávila RC. Formação das mulheres nas escolas de Medicina. Rev Bras Educ Med. 2014;38(1):142-9 [acesso em 13 mar 2021]. Disponível em: http://www.scielo.br/scielo.php?script=sci_arttext\&pid=S010055022014000100019\&lng=en\&nrm=iso\&tlng=pt.
12. Allen I. Women doctors and their careers: What now? BMJ. 2005;331(7516):569-72 [acesso em 13 mar 2021]. Disponível em: https:// www.bmj.com/content/331/7516/569.

13. Silva TD. Ação afirmativa e população negra na educação superior: acesso e perfil discente. Rio de Janeiro: Ipea; 2020 [acesso em 20 mar 2021]. Disponível em: https://www.ipea.gov.br/portal/images/stories/PDFs/TDs/ td_2569.pdf.

14. Cardoso Filho FAB, Magalhães JF, Silva KML, Pereira ISSD. Perfil do estudante de Medicina da Universidade do Estado do Rio Grande do Norte (UERN), 2013. Rev Bras Educ Med. 2015;39(1):32-40. doi: 10.1590/1981-52712015v39n1e01092014.

15. Veras RM, Fernandez CC, Feitosa CCM, Fernandes S. Perfil socioeconômico e expectativa de carreira dos estudantes de Medicina da Universidade Federal da Bahia. Rev Bras Educ Med. 2020;44(2):e056. doi: 10.1590/19815271v44.2-20190208.

16. Pirotta KCM, Schor N. Reproductive intentions and fertility regulation practices among university students. Rev Saude Publica. 2004;38(4):495502 [acesso em 13 mar 2021]. Disponível em: http://www.fsp.usp.br/rsp.

17. Araújo J, Bacelar S, Jesus LE. Family planning among female medical students: are their plans comparable to other professionals? Rev Assoc Med Bras. 2020;66(4):485-90. doi: 10.1590/1806-9282.66.4.485.

18. Scavone L. Maternidade: transformações na família e nas relações de gênero. Interface Comun Saúde Educ. 2001;5(8):47-60.

19. Mattar LD, Grilo CSD. Hierarquias reprodutivas: maternidade e desigualdades no exercício de direitos humanos pelas mulheres. Interface Comun Saúde Educ. 2012;16(40):107-19.

20. Buddeberg-Fischer B, Stamm M, Buddeberg C, Bauer G, Hämmig O, Knecht $M$, et al. The impact of gender and parenthood on physicians' careers professional and personal situation seven years after graduation. BMC Health Serv Res. 2010;10(40):1-10 [acesso em 13 mar 2021]. Disponível em: http://www.biomedcentral.com/1472-6963/10/40.

21. Buddeberg-Fischer B, Stamm M. The medical profession and young physicians' lifestyles in flux: challenges for specialty training and health care delivery systems. Swiss Med Wkly. 2010;140:1-9 [acesso em 13 mar 2021]. Disponível em: http://www.smw.ch.

22. Rocha G, Tagma B, Donelli MS. Maternity and career: challenges forward to reconciliation of roles. Aletheia. 2012;38(39):206-17.

23. Ribeiro MMF, Leal SS, Diamantino FC, Bianchi HA. A opção pela Medicina e os planos em relação ao futuro profissional de estudantes de uma faculdade pública brasileira. Rev Bras Educ Med. 2011;35(3):405-11 [acesso em 13 mar 2021]. Disponível em: http://www.scielo.br/scielo. php?script=sci_arttext\&pid=S0100-55022011000300015\&lng=en\&nrm=i so\&tlng=pt.

24. Instituto Nacional de Estudos e Pesquisas Educacionais Anísio Teixeira Relatório síntese de área: Medicina. Brasília: Inep; 2019 [acesso em 8 jul 2021]. Disponível em: https://download.inep.gov.br/educacao_superior/ enade/relatorio_sintese/2019/Enade_2019_Relatorios_Sintese_Area_ Medicina.pdf. 